\title{
Le World Hospital Pharmacy Research Consortium - Suivi de la pratique dans le monde relativement aux déclarations de Bâle
}

\author{
par Rebekah Moles, Betty Chaar et Jonathan Penm
}

$\mathrm{L}$ es pharmaciens d'hôpitaux détiennent un rôle unique et important en santé qui, d'ailleurs, a une portée considérable sur les résultats thérapeutiques. Toutefois, la majorité des articles publiés s'appuient sur des modèles de pratique de la pharmacie hospitalière des pays développés.

Les déclarations de Bâle représentent le résultat d'une conférence mondiale inaugurale à Bâle en Suisse, en 2008, organisée par la Section de pharmacie hospitalière de la Fédération internationale pharmaceutique. Ces déclarations consensuelles mondiales traduisent la vision de l'avenir pour la pharmacie hospitalière que privilégient les membres de la profession ${ }^{1}$. Actuellement, les 75 déclarations (abordant toutes les sphères du processus de distribution des médicaments à l'hôpital, notamment l'approvisionnement en médicaments, les influences sur la prescription, la préparation et la délivrance des médicaments, l'administration des médicaments, la surveillance des pratiques relatives aux médicaments ainsi que les ressources humaines et la formation) font l'objet de mises à jour mineures, qui doivent être terminées cette année. D’autre part, les principes généraux de sécurité des médicaments et de pratiques fondées sur les données probantes demeureront inchangés.

Cependant, les déclarations et les lignes directrices ne sont pertinentes que lorsqu'elles se retrouvent dans la pratique et seulement lorsque les personnes et les organisations s'efforcent de satisfaire aux critères qu'elles contiennent. D'ailleurs, il est admis que le suivi des pratiques représente un élément essentiel de l'assurance de la qualité de même que le seul moyen de vérifier que les interventions parviennent à améliorer une situation. C'est pourquoi le World Hospital Pharmacy Research Consortium (WHoPReC) a été mis sur pied pour surveiller les pratiques de pharmacie hospitalière dans le monde relativement aux déclarations de Bâle².

Ce numéro du JCPH, comme d'autres numéros, est fortement orienté sur le contenu des déclarations de Bâle. Par exemple, Mackenzie et ses collaborateurs ${ }^{3}$ ont découvert que le recours à une posologie non standard de nimodipine pour traiter l'hémorragie sous-arachnoïdienne anévrysmale est lié à un vasospasme. Cette étude donne aux pharmaciens des arguments fondés sur des données probantes leur permettant d'instruire les médecins et les autres membres du personnel hospitalier à propos des bonnes pratiques d'utilisation des médicaments. À ce titre, elle soutient la $14^{\mathrm{e}}$ déclaration de Bâle, qui dit : "Les pharmaciens d'hôpitaux doivent initier et former le personnel infirmier, les médecins et les autres membres du personnel hospitalier quant aux bonnes pratiques d'utilisation des médicaments $»^{1}$. En outre, la $56^{\text {e }}$ déclaration de Bâle ( Les pharmaciens d'hôpitaux doivent veiller à l'élaboration de stratégies d'assurance de la qualité relatives à l'administration des médicaments, notamment l'emploi d'une méthode d'observation permettant de repérer les erreurs et de dégager les priorités en matière d'amélioration " ${ }^{1}$ ) a servi de base pour le travail de Mackenzie et de ses collaborateurs ${ }^{3}$ au cours de leur recherche. D'autres déclarations de Bâle sous le thème " administration " sont actuellement à l'étude par des membres du WHoPReC des Amériques; des interviews qualitatives, menées auprès de pharmaciens de l'Amérique du Nord et du Sud, ont pour objectif de cerner les déclarations qui posent problèmes aux pharmaciens d'hôpitaux, et ceci, pour que des stratégies d'interventions puissent faire l'objet d'essais futurs.

Le problème de pénuries de médicaments, un sujet d'actualité, est aussi abordé dans les déclarations de Bâle, car les pénuries touchent les pays développés au même titre que les pays en développement. La déclaration 25 stipule : "Chaque pharmacie doit disposer de plans d'intervention pour l'approvisionnement en médicaments dans l'éventualité de pénurie ou d'urgence »" ${ }^{1}$. Dans ce numéro, Barthélémy et ses collaborateurs ${ }^{4}$ présentent un nouveau compte rendu des pénuries de médicaments au Canada entre septembre 2012 et août 2013 en comparant les données de cette période à celles entre 2006 et 2012. Bien qu'ils indiquent qu'il y a eu moins de pénuries de médicaments en 2012 - 2013, des préoccupations subsistent en ce qui a trait à la qualité et à la 
sécurité des médicaments produits à l'étranger. Barthélémy et ses collaborateurs ${ }^{4}$ recommandent que tous les hôpitaux conservent l'équivalent de 60 jours d'un stock de sécurité de médicaments essentiels afin de pallier les éventuelles conséquences de pénuries de médicaments. De telles recommandations permettent de s'assurer que les pharmaciens d'hôpitaux respectent les standards des déclarations de Bâle. Comme les pénuries de médicaments touchent des patients de partout dans le monde, le WHoPReC a aussi amorcé des recherches sur ce sujet. Une étudiante canadienne de cycle supérieur, qui termine un diplôme de recherche à l'Université de Sydney, à Sydney en Australie, s'est penchée sur les éléments complexes de la chaîne d'approvisionnement et elle a étudié les vulnérabilités qui y sont présentes selon le point de vue des principaux leaders d'opinion. Elle a examiné le concept de la « liste des médicaments essentiels ", un outil largement utilisé dans les pays en développement, pour savoir s'il peut être utile aux pays développés comme l'Australie et le Canada. Une étudiante népalaise, se trouvant aussi à l'Université de Sydney, s'intéresse aux déclarations de Bâle sous le thème de l'approvisionnement et elle accorde une attention particulière à l'approvisionnement éthique, souvent un enjeu dans les pays en développement comme le Népal.

Bien que les pharmaciens d'hôpitaux doivent conserver leurs responsabilités sur le plan de l'approvisionnement de médicaments, nombreux sont ceux qui ont aussi endossé un rôle plus clinique qui influence l'acte de prescription. Sept des déclarations de Bâle font la promotion de l'influence qu'ont les pharmaciens d'hôpitaux sur l'acte de prescription, notamment la $32^{\mathrm{e}}$ déclaration qui indique qu'une "formation clinique postuniversitaire devrait être organisée afin de préparer les pharmaciens hospitaliers à prescrire les médicaments en collaboration... $"^{1}$. Toutefois, les pharmaciens, tout comme leurs collègues médecins, courent le risque de faire des prescriptions inadéquates. La chronique « Le pour et le contre ${ }^{5,6} \mathrm{du}$ présent numéro fait ressortir les avantages et les désavantages de la prescription de médicaments par les pharmaciens d'hôpitaux. Peu importe les opinions des lecteurs à savoir si les pharmaciens devraient ou non prescrire des médicaments, on ne peut nier que ces professionnels de la santé ont une influence sur l'acte de prescrire. D'ailleurs, le WHoPReC a réalisé plusieurs études sur le sujet dans la région du Pacifique occidental, car beaucoup de pays en Asie commencent à mettre en place des services de pharmacie clinique dans leurs hôpitaux ${ }^{7}$. De telles recherches ont mené au développement d'outils de recherche validés permettant d'observer l'influence qu'ont les pharmaciens d'hôpitaux sur l'acte de prescription dans 37 pays $^{7-9}$. Ces recherches ont aussi mis en relief que, bien que les compétences et la motivation des pharmaciens d'hôpitaux soient nécessaires à la mise en œuvre de nouveaux services, les différents appuis des administrateurs d'hôpitaux, des médecins et des patients sont cruciaux pour la réussite de ces services ${ }^{10,11}$. Ainsi, des études axées sur l'obtention d'appuis à l'égard de l'influence des pharmaciens d'hôpitaux sur l'acte de prescription sont essentielles, pour des raisons régionales et internationales.

Comme le WHoPReC cherche à produire des études à caractère international de grande qualité sur les pratiques de la pharmacie hospitalière afin d'améliorer les résultats en santé, d'autres recherches sont menées ${ }^{2}$. La portée des recherches s'est élargie afin d'inclure d'autres thèmes des déclarations de Bâle dans d'autres régions du monde. Par exemple, des chercheurs du WHoPReC de l'Afrique se penchent sur la préparation et la délivrance des médicaments, alors que dans la région orientale de la Méditerranée, l'accent est mis sur les ressources humaines et la formation.

Des recherches de partout dans le monde continuent de montrer que les déclarations de Bâle sont pertinentes pour les pays développés et en développement. Le travail du WHoPReC a mis en relief les sphères de la pratique de la pharmacie hospitalière qui nécessitent toujours des améliorations, et ce, peu importe le statut de développement du pays. Nous encourageons les autres chercheurs qui s'intéressent à la pharmacie hospitalière internationale à communiquer avec le WHoPReC afin de participer à ce mouvement. La mondialisation et les attentes plus élevées des patients font en sorte que chaque pharmacien d'hôpital doit aller chercher à l'étranger des moyens novateurs de pratiquer sa profession. Nous espérons que le WHoPReC aidera à faciliter les progrès internationaux de la pratique de la pharmacie hospitalière afin d'améliorer les résultats thérapeutiques partout dans le monde.

\section{Références}

1. The Basel Statements on the future of hospital pharmacy. Am J Health Syst Pharm. 2009;66(5 Suppl 3):S61-6.

2. World Hospital Pharmacy Research Consortium [site Web]. Sydney (Australie) : University of Sydney; 2014. Publié au : www.whoprec.org. Consulté le 13 août 2014 .

3. MacKenzie M, Gorman SK, Doucette S, Green R. Incidence of and factors associated with manipulation of nimodipine dosage in patients with aneurysmal subarachnoid hemorrhage. Can J Hosp Pharm. 2014;67(5):358-65.

4. Barthélémy I, Lebel D, Bussières JF. Drug shortages in health care institutions: perspectives in early 2014. Can J Hosp Pharm. 2014;67(5):387-9.

5. Mansell H. Should hospital pharmacists prescribe? The "pro" side. Can J Hosp Pharm. 2014;67(5):390-2.

6. Hopf Y. Should hospital pharmacists prescribe? The "con" side. Can J Hosp Pharm. 2014;67(5):392-3.

7. Penm J, Chaar B, Rose G, Moles R. Pharmacists' influences on prescribing: validating a clinical pharmacy services survey in the Western Pacific Region. Res Social Adm Pharm. 19 juin 2014. doi: 10.1016/j.sapharm.2014.04.001 (diffusion en ligne avant impression).

8. Penm J, Chaar B, Moles R. Developing a validated survey to assess the Basel Statements: the Western Pacific Region's response. Int Pharm J. 2010; 26(2):34-8

9. Penm J, Chaar B, Moles R. Validating a hospital medicines formulary survey in the Western Pacific Region — a global hospital pharmacy initiative based on the Basel Statements. Res Social Adm Pharm. 2012;8(4):298-308.

10. Penm J, Chaar B, Dechun J, Moles R. Formulary systems and pharmacy and therapeutics committees in the Western Pacific Region: exploring two Basel Statements. Am J Health Syst Pharm. 2013;70(11):967-79. 
11. Penm J, Moles R, Wang H, Li Y, Chaar B. Factors affecting the implementation of clinical pharmacy services in China. Qual Health Res. 2014; 24(3):345-56.

Rebekah J Moles, B. Pharm., Dip. Hosp. Pharm., Ph. D., Grad. Cert. Ed. Stud. (Higher Ed.), travaille à la Faculté de pharmacie de I'Université de Sydney, Sydney, Australie. Elle est également rédactrice adjointe du JCPH.

Betty Chaar, B. Pharm., MHL, Ph. D., travaille à la Faculté de pharmacie de I'Université de Sydney, Sydney, Australie.
Jonathan Penm, B. Pharm. (Hons.), travaille à la Faculté de pharmacie de l'Université de Sydney, Sydney, Australie.

Intérêts concurrents : Les auteurs sont des membres fondateurs du World Hospital Pharmacy Research Consortium, un réseau mondial indépendant de chercheurs dirigé par des bénévoles. Aucun autre intérêt concurrent déclaré.

\section{Adresse de correspondance :}

De Rebekah J Moles

Faculty of Pharmacy

The University of Sydney

Pharmacy and Bank Building A15

Sydney NSW 2006 Australia

Courriel : rebekah.moles@sydney.edu.au

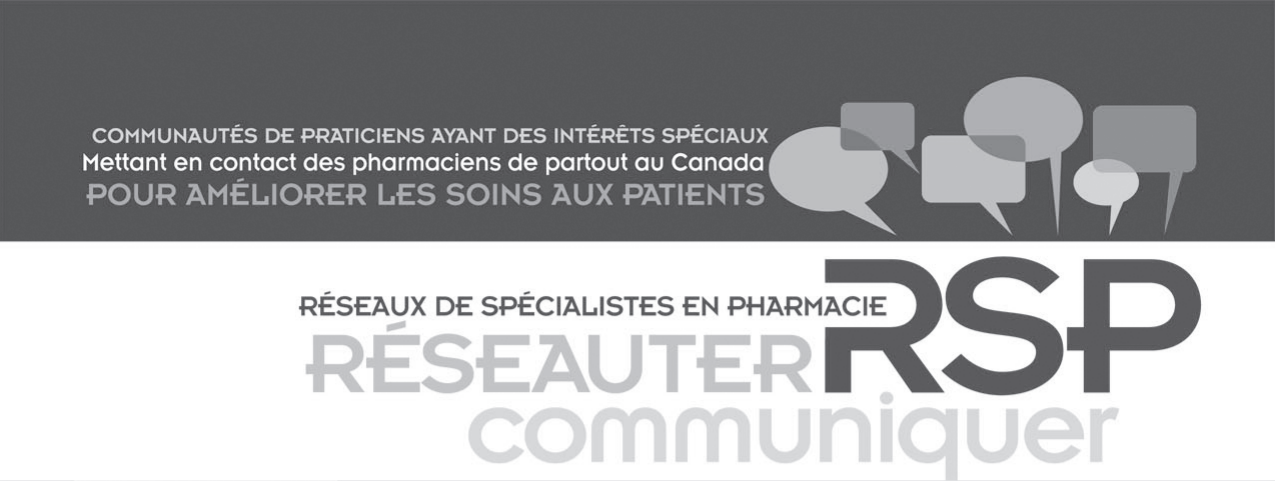

La SCPH compte plus de 20 RSP auxquels vous pouvez participer! Visitez le www.cshp.ca pour la liste complète.
Participez aux Réseaux de spécialistes en pharmacie! Les membres de la SCPH vous mettent en contact avec ce qui est important : des gens et de l'information.

\section{Les RSP :}

- mettent les membres en contact avec d'autres personnes qui ont une passion pour un aspect particulier de la profession de pharmacien

- facilitent le partage rapide d'idées, de développements, de méthodes, d'expériences, de connaissances pour améliorer la pratique

- favorisent la collaboration à des projets, à des recherches et à des programmes éducatifs pour répondre aux besoins des membres des RSP

- proposent des occasions supplémentaires aux membres d'agir à titre de leaders d'opinion et de ressources clés pour le Conseil de la SCPH sur des questions de pratique spécialisée, dont la rédaction de déclarations de principes, de lignes directrices et des documents d'information pertinents

La participation aux RSP est gratuite pour les membres de la SCPH.

Visitez MY.CSHP.ca et inscrivez-vous dès aujourd'hui!

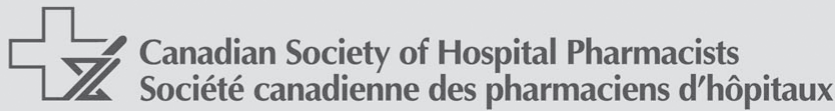

